\title{
Investigation into the acute effects of intermittent energy restriction on postprandial substrate metabolism
}

\author{
R. Antoni ${ }^{1}$, K.L. Johnston ${ }^{2}$, A. Collins ${ }^{1}$ and M.D. Robertson ${ }^{1}$ \\ ${ }^{1}$ Nutrition, Metabolism and Diabetes Research Group, Faculty of Health and Medical Sciences, University of Surrey, \\ Guildford, UK and ${ }^{2}$ Lighterlife UK Ltd, Cavendish House, Parkway, Harlow Business Park, Essex, UK
}

\section{This abstract was awarded the student prize for best oral original communication}

The intermittent energy restriction (IER) approach to weight-loss involves short periods of substantial energy restriction (ER) followed by non-restricted intake ${ }^{(1)}$. Little is known about the acute effects of total and partial ER on postprandial substrate metabolism within overweight/obese populations, which forms the main objective of this study. Secondary outcomes included subsequent energy compensation.

Ten (three female) healthy, overweight/obese $\left(36 \pm 5 \mathrm{y} ; 29.0 \pm 1 \cdot 1 \mathrm{~kg} / \mathrm{m}^{2}\right)$ subjects were recruited into this three-way, cross-over dietary investigation. Subjects completed three one-day dietary interventions in a randomized order with a one-week washout: isoenergetic intake, partial $75 \%$ ER (using LighterLife ${ }^{\mathrm{TM}}$ FoodPacks) and total $100 \%$ ER. Postprandial responses to a liquid test-meal were assessed the following day via serial blood measurements. Subjects also completed dietary diaries for two subsequent days of ad libitum intake. Data were analysed using repeated measures ANOVA and presented as mean \pm SEM.

Relative to the isoenergetic control leg, postprandial glucose tended to be higher after partial ER ( $p=0.089)$, and was significantly increased following total ER with a delay in the time to peak (both $p<0.05$ ) (Fig 1A). Postprandial triacylglycerol was reduced after partial and total ER, by 22 and 39\% respectively (both p<0.05) (Fig 1B). Fasting and postprandial hepatic production of 3- $\beta$-hydroxybutyrate $(3-\beta \mathrm{OHB})$ were elevated after both ER interventions (both $p<0.05)$ (Fig 1C). Cumulative 3-day energy intakes remained significantly lower after both total and partial ER interventions, with subjects sustaining comparable energy deficits of $-28 \pm 5 \%$ and $-30 \pm 3 \%$ respectively (all $\mathrm{p}<0.001$ vs iso).
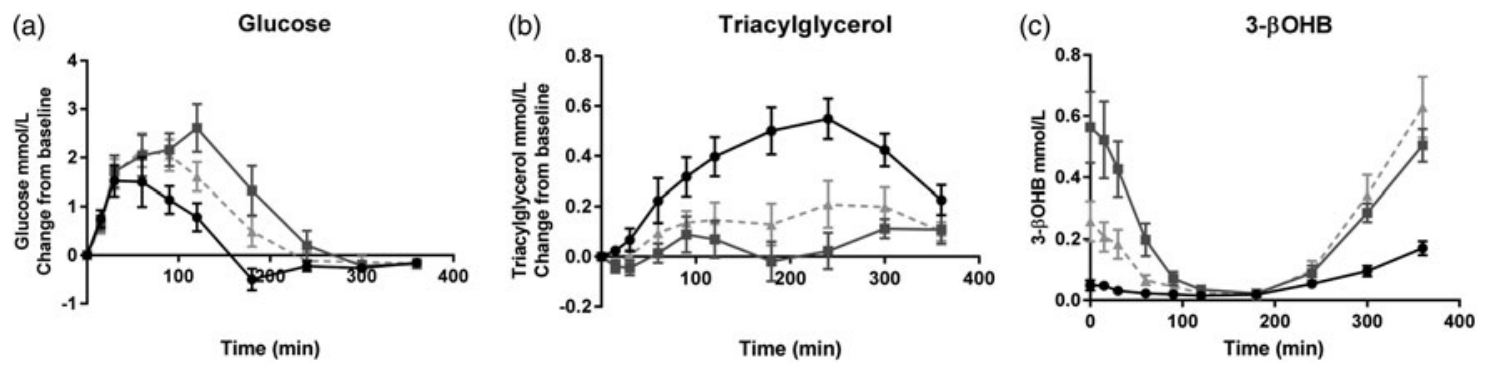

Figure 1A-C. Postprandial substrate responses

Total $100 \%$ ER (- $)$, partial $75 \%$ ER $(-*)$, isoenergetic control diet

One day of substantial (75-100\%) ER was sufficient to produce acute improvements in postprandial triacylglycerol, which we hypothesise was driven by a shift in hepatic fatty-acid partitioning towards oxidation. By allowing some food intake, partial ER was able to mitigate the increase in postprandial glycaemia found with total fasting whilst furthermore producing a comparable threeday energy deficit. Findings of this acute study highlight the potential utility of IER as a treatment strategy for hyperlipidaemia, but now requires translation over chronic timescales.

Antoni R, Johnston K, Collins A and Robertson MD (2014) Res Endocrinol. 2014 Article ID: 459119 